\title{
Studies on the Detoxicating Hormone of the Liver (Yakriton).
}

\author{
95th Report. \\ Lowering of Detoxicating Liver Power in Hot Seasons. \\ $\mathrm{By}$ \\ Akira Sato and Fumio Ohta. \\ (经藤彰) (太王文雄) \\ (From the Department, of Pediatrics, Faculty of Medicine, \\ Tohoku Imperial University, Sendai. \\ Director: Prof. A. Sato.)
}

\section{Introduction.}

Among clinicians of different specialities, it is probably pediatricians who think most of the injury of heat upon the human being. Epidemic acidosis or infantile diarrhoea would not occur at all, or, even if it does, only mildly, without the summer heat. And the injury is done to the whole organism, but it is not dificult to presume that the liver is the organ, or at least one of the organs-damaged most severely. Sakurada and Sato (one of us) devised Sato and Sa$\mathrm{k}$ urada' $\mathrm{s}^{1)}$ liver function test of rabbits. The test consists of an intraperitoneal injection of $3 \%$ ammonium chloride in an amount of 10 c.c. per kilo of body weight. Rabbits put to the test will manifest a symptom according to their liver power of ammonia detoxication, as is shown in Table 1.

It has been the experience of Sato (one of us) that in summer time rabbits are apt to become of a lower class liver power. But no systematic experiment has been performed so far, consequently we desire to report of a systematic trial concerning the problem in the present paper.

1) A. S a to and H. Sakurad a, Tohoku J. Exp. Med, 1927, 8, 347. 
TABLe 1.

Classification of detoxicating power of animals.

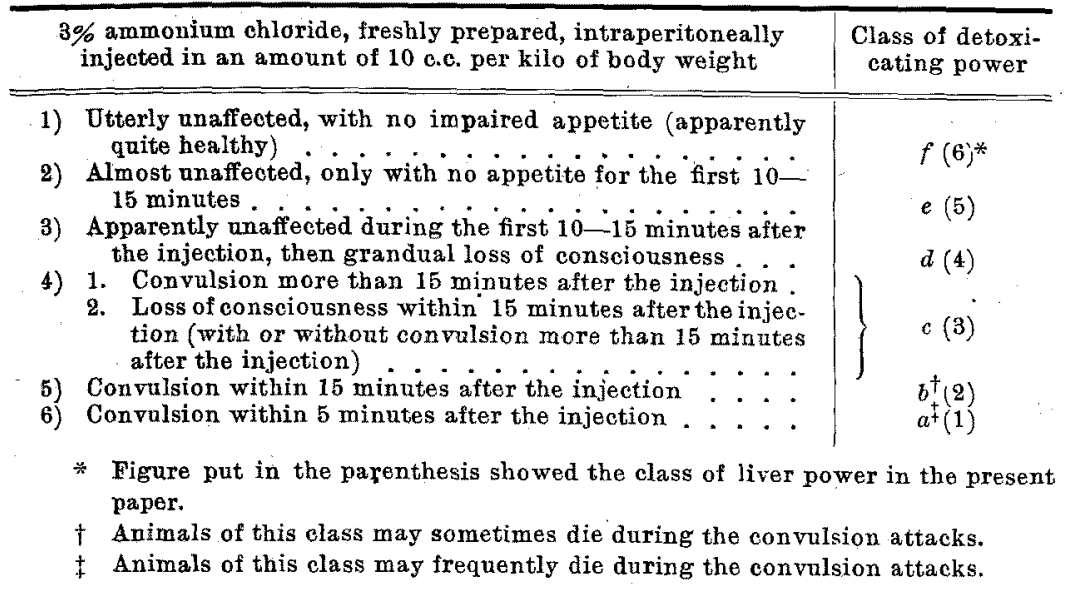

\section{Method of Experiment.}

1. Healthy rabbits fed on tofukara were used.

A number of them were placed in the animal house for a certain length of time (about one week) and then tested with $S$ ato and $S$ akurada's liver test 3 times. Then all of them were transferred into the cellar, where they stayed about one week, and then tested again with $\mathrm{Sato}$ and $\mathrm{Sakurad} \mathrm{a}^{\prime} \mathrm{S}^{1}$ liver function test. Again they were placed in the animal house about one week and then tested with $S$ ato and $S$ akurad a's $\mathbf{S}^{17}$ liver test. And so on.

In another series of experiments, the following method was used. While one group of the animals was kept in the cellar and tested with the ammonium injection, the other group was kept in the animal house and tested with the ammonium injection. Then the former group was transferred into the animal house and the latter into the cellar. And so on.

2. Sato and Sakurada's liver test."

As stated above, each group of rabbits stayed about a week in the animal house or in the cellar respectively, for the purpose of-so to speak-" acclimatisation". Then the liver test was performed three times. The interval between each two tests was one or two days.

\section{Presumption of Result.}

The animal house, as above stated, was on the ground, and the wall was thin; the cellar was half-way under the ground and the wall 
was thick. Accordingly, the temperature fluctuation in summer* was appreciably wider in the anima house than in the cellar, though in the other seasons the fluctuation was not so remarkable.

Now our presumption of the result was like this :-

When rabbits were placed in the cellar in summer, they would bear themselves toward ammonium injection like animals with more or less high-classed liver power, or they would not be severely affected in spite of the ammonium injection.

But when they were placed in the animal house, they now would bear themselves like animals with more or less low-classed liver power, or they would be affected more or less severely by the ammonium injection. In the other seasons the result would show the same inclination, though it might not be so remarkable as in summer.

Or, if shown in a small table, the presumed result would be simply like the following :-

$\begin{array}{llll}\text { Cellar } & \text { Animal house } & \text { Cellar } & \text { Animal house } \\ \text { High-classed } & \text { Low-classed } & \text { High-classed } & \text { Low-classed }\end{array}$

This was our presumption of the result. Now the actual result will be related.

\section{Actual Result.}

The actual result of the present experiment is shown in Tables 2, $3 \mathrm{~A}, 3 \mathrm{~B}, 4 \mathrm{~A}$ and $4 \mathrm{~B}$. And the evident result will be seen from Figures 1,2 A, $2 \mathrm{~B}, 2 \mathrm{~A}$ and $3 \mathrm{~B}$.

All the Figures show that Point $\mathrm{C}$ was generally higher than the preceding or the following Point A. This shows that, when the rabbits were kept in the cellar, their liver showed a stronger ammonia detoxicating ability, while, when kept in the animal house, they generally showed a lower-classed liver power.

Fig. 1. Experiment in artumn.

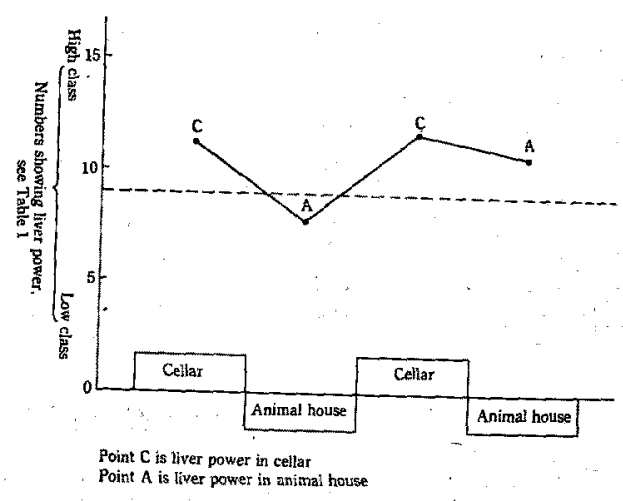

* In winter too (See comment). 
Fig: 2a. The 1st experiment in winter.

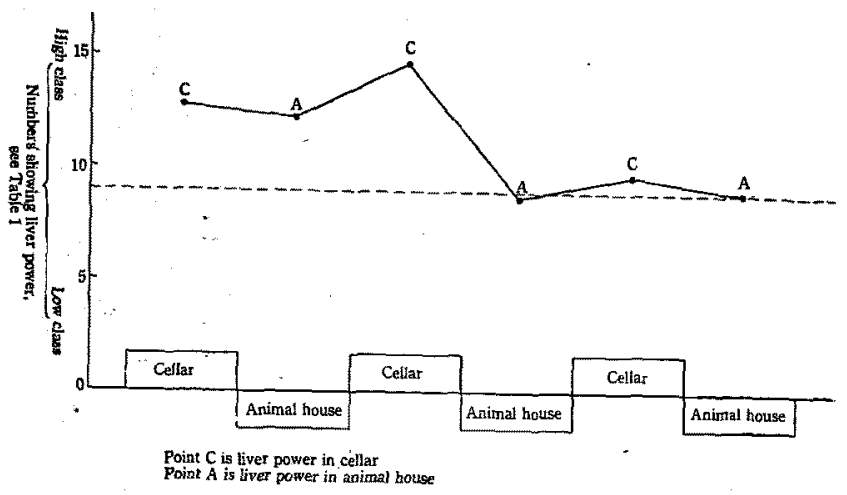

Fig. 2b. The 2 nd experiment in winter.

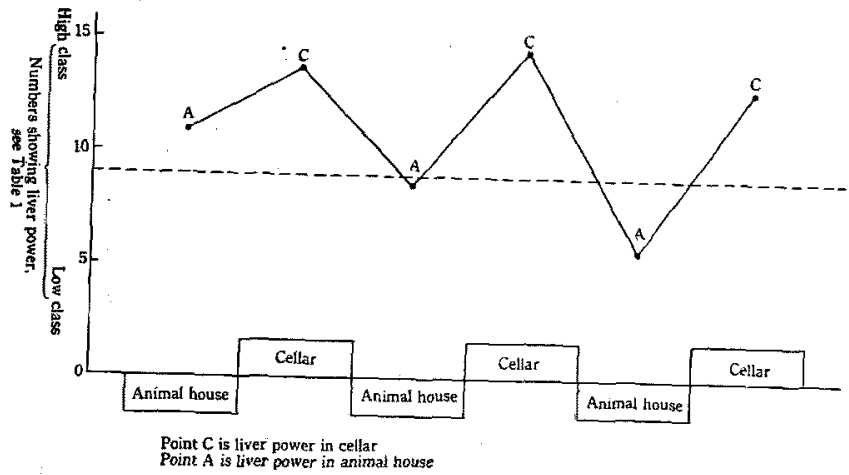

This result actually obtained was on the whole coincident with what had been presumed above, but different from the presumption in smaller details. We shall come upon this problem in the Comment. 

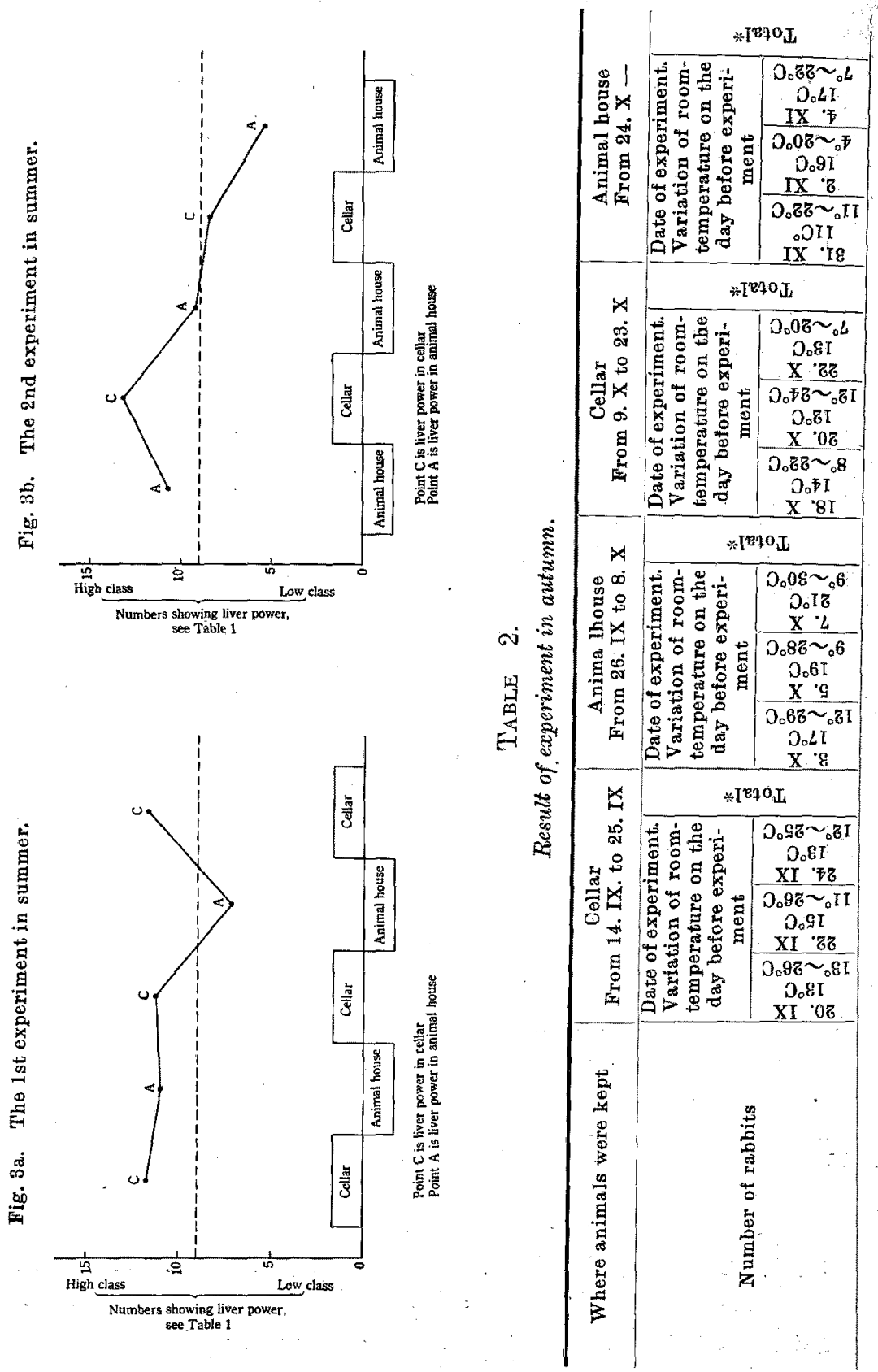


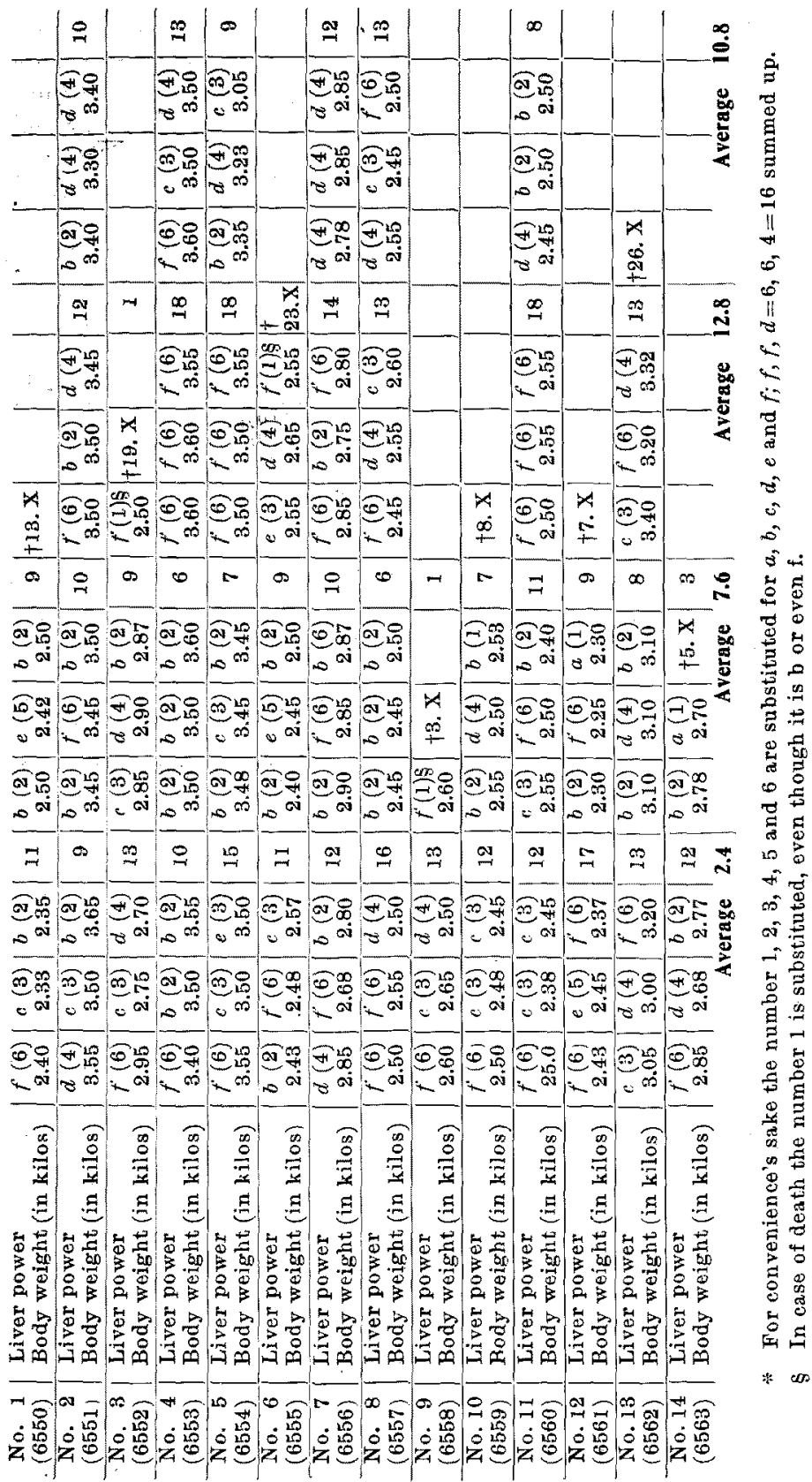


(A)

TABLE

Result of the 1st experi-

\begin{tabular}{|c|c|c|c|c|c|c|c|c|c|c|}
\hline \multirow{3}{*}{$\begin{array}{c}\text { Where animals were kept } \\
\text { Number of rabbits }\end{array}$} & \multicolumn{4}{|c|}{$\begin{array}{l}\text { Cellar } \\
\text { From 4. I to } 15.1 \\
\end{array}$} & \multicolumn{4}{|c|}{$\begin{array}{l}\text { Animal house } \\
\text { From } 16.1 \text { to } 29.1\end{array}$} & \multirow{2}{*}{\multicolumn{2}{|c|}{\begin{tabular}{|} 
Cellar \\
Form 30.1 \\
Date of ex- \\
ment. Va- \\
tion of \\
tempera- \\
on the day \\
fore experi-
\end{tabular}}} \\
\hline & \multicolumn{3}{|c|}{$\begin{array}{l}\text { Date of experi- } \\
\text { ment. Variation } \\
\text { of room-tem- } \\
\text { perature on the } \\
\text { day before } \\
\text { experiment }\end{array}$} & \multirow{2}{*}{ 害 } & \multicolumn{3}{|c|}{$\begin{array}{l}\text { Date of experi- } \\
\text { ment. Variation } \\
\text { of room-tem- } \\
\text { perature on the } \\
\text { day before } \\
\text { experiment }\end{array}$} & \multirow{2}{*}{$\frac{\frac{1}{6}}{5}$} & & \\
\hline & 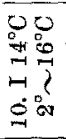 & 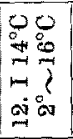 & $\left|\begin{array}{cc}0 & 0 \\
0 & 0 \\
\cos & 0 \\
-1 & -1 \\
1 & \gamma \\
0 & 0 \\
7 & 0\end{array}\right|$ & & 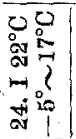 & $\mid \begin{array}{cc}0 & 0 \\
0 & 0 \\
0 & 0 \\
- & 3 \\
1 & ? \\
0 & 0 \\
0 & 0\end{array}$ & {$\left[\begin{array}{ll}0 & 0 \\
0 & 0 \\
0 & 0 \\
0 & 3 \\
-1 & 3 \\
\infty & 0 \\
0 & 1\end{array}\right]$} & & $\begin{array}{ll}0 & 0 \\
0 & 0 \\
-1 & 0 \\
1 & 0 \\
1 & 2 \\
10 & 0\end{array}$ & 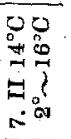 \\
\hline 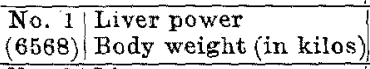 & $\begin{array}{l}f(6) \\
3.30 \\
\end{array}$ & $\begin{array}{l}d(4) \\
3.20 \\
\end{array}$ & $\begin{array}{l}d(4) \\
3.25 \\
\end{array}$ & 14 & $\begin{array}{l}7(6) \\
8.15\end{array}$ & $\begin{array}{l}7(6) \\
3.30\end{array}$ & $\begin{array}{r}t^{2}(6) \\
3.25 \\
\end{array}$ & 18 & $\begin{array}{l}c(3) \\
3.25 \\
\end{array}$ & \\
\hline $\begin{array}{l}\text { No. 2 Liver power } \\
(6569,) \text { Body weight (in kilos) }\end{array}$ & $\begin{array}{l}f(6) \\
2.30 \\
\end{array}$ & $\begin{array}{l}e(5) \\
2.25 \\
\end{array}$ & $\begin{array}{l}f(6) \\
2.25\end{array}$ & 17 & $\begin{array}{l}d(4) \\
2.38\end{array}$ & $\begin{array}{l}c(3) \\
2.47 \\
\end{array}$ & $\begin{array}{l}e(3) \\
2.48 \\
\end{array}$ & 10 & $\begin{array}{l}f(6) \\
2.50\end{array}$ & $\begin{array}{l}f(6) \\
2.40\end{array}$ \\
\hline \begin{tabular}{l|l|}
$\begin{array}{l}\text { No. } \\
(6570,\end{array}$ & Liver power \\
\end{tabular} & $\begin{array}{l}f(6) \\
2.87 \\
\end{array}$ & $\begin{array}{l}f^{\prime}(6) \\
2.80 \\
\end{array}$ & $\begin{array}{l}f(6) \\
2.77 \\
\end{array}$ & 18 & $\begin{array}{l}6(3) \\
2.65 \\
\end{array}$ & $\begin{array}{l}f(6) \\
2.65 \\
\end{array}$ & $\begin{array}{l}f(1) 8 \\
2.75 \\
\end{array}$ & 10 & $\mathbf{I}$ & \\
\hline $\begin{array}{l}\text { No. } 4 \text { Liver power } \\
(6571) \text { Body weight (in kilos) }\end{array}$ & $\begin{array}{l}e(5) \\
3.35 \\
\end{array}$ & $\begin{array}{l}f(6) \\
3.42 \\
\end{array}$ & $\begin{array}{l}f(6) \\
3.38 \\
\end{array}$ & 17 & $\begin{array}{l}c(3) \\
3.65\end{array}$ & $\begin{array}{l}7(6) \\
3.65\end{array}$ & $\begin{array}{l}f(6) \\
3.75 \\
\end{array}$ & 15 & $\begin{array}{l}f(6) \\
3.57 \\
\end{array}$ & $\begin{array}{l}d(4) \\
3.57 \\
\end{array}$ \\
\hline $\begin{array}{l}\text { No. } 5 \text { Liver power } \\
(6572, \text { Body weight (in kilos) }\end{array}$ & $\begin{array}{l}d(4) \\
3.40\end{array}$ & $\begin{array}{l}f(6) \\
3.20 \\
\end{array}$ & $\begin{array}{l}d(4) \\
3.30 \\
\end{array}$ & 14 & $\begin{array}{l}e(b) \\
3.27\end{array}$ & $\begin{array}{l}d(4) \\
3.20 \\
\end{array}$ & $\begin{array}{l}e(5) \\
3.25 \\
\end{array}$ & 12 & $\begin{array}{l}f(6) \\
3.25 \\
\end{array}$ & $\begin{array}{l}d(4) \\
3.25 \\
\end{array}$ \\
\hline \begin{tabular}{l|l} 
No. 6 & Liver power \\
$(6573)$ & Body weight (in kilo
\end{tabular} & $\begin{array}{l}\bar{d}(4) \\
3.15\end{array}$ & $\begin{array}{l}d(4) \\
3.20 \\
\text { Aver }\end{array}$ & $\begin{array}{l}e(5) \\
3.10 \\
\text { ge }\end{array}$ & 13 & $\begin{array}{l}c(3) \\
3.17\end{array}$ & $\begin{array}{l}c(3) \\
3.25 \\
\text { Aver }\end{array}$ & $\begin{array}{l}c(3) \\
3.25 \\
\text { age }\end{array}$ & 3 & $\begin{array}{l}c(3) \\
3.10\end{array}$ & $\begin{array}{l}e(5) \\
3.15\end{array}$ \\
\hline
\end{tabular}

Result of the 2nd experi-

\begin{tabular}{|c|c|c|c|c|c|c|c|c|c|c|}
\hline Where animals were kept & \multicolumn{4}{|c|}{$\begin{array}{l}\text { Animal house } \\
- \text { to } 15 . \mathrm{I}\end{array}$} & \multicolumn{4}{|c|}{$\begin{array}{l}\text { Cellar } \\
\text { From 16.I to 29. I }\end{array}$} & \multicolumn{2}{|c|}{$\begin{array}{r}\text { Animal } \\
\text { From } 30.1\end{array}$} \\
\hline \multirow[t]{2}{*}{ Number of rabbits } & \multicolumn{3}{|c|}{$\begin{array}{l}\text { Date of experi- } \\
\text { ment Variation } \\
\text { of room-tem- } \\
\text { perature on the } \\
\text { day before } \\
\text { experiment }\end{array}$} & \multirow{2}{*}{ 雚 } & \multicolumn{3}{|c|}{$\begin{array}{l}\text { Date of experi- } \\
\text { ment Variation } \\
\text { of room-tem- } \\
\text { perature on the } \\
\text { day before } \\
\text { experiment }\end{array}$} & \multirow{2}{*}{ 密 } & \multicolumn{2}{|c|}{$\begin{array}{r}\text { Date of ex- } \\
\text { ment Va- } \\
\text { tion of } \\
\text { tempera- } \\
\text { on the day } \\
\text { fore experi- }\end{array}$} \\
\hline & $\begin{array}{cc}0 & 0 \\
0 & 0 \\
0 & 00 \\
-1 & 7 \\
1 & 3 \\
0 & 0 \\
0 & 0\end{array}$ & 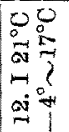 & 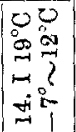 & & 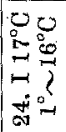 & 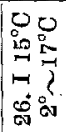 & $\mid \begin{array}{ll}0 & 0 \\
10 & 0 \\
2 & 0 \\
w & 2 \\
\infty & 0 \\
a & 0\end{array}$ & & $\begin{array}{ll}0 & 0 \\
0 & 0 \\
0 & 10 \\
0 & = \\
= & 0 \\
0 & 0 \\
0 & 0\end{array}$ & 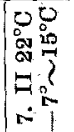 \\
\hline \begin{tabular}{l|l|} 
No. 7 & Liver power \\
$(6574)$ & Body weight (in kilos)
\end{tabular} & $\begin{array}{l}f(6) \\
3.10\end{array}$ & $\begin{array}{l}c(3) \\
3.10\end{array}$ & $\begin{array}{l}5(2) \\
3.05\end{array}$ & 11 & $\begin{array}{l}e(5) \\
3.10\end{array}$ & $\begin{array}{l}b(2) \\
3.00\end{array}$ & $\begin{array}{l}d(4) \\
2.95\end{array}$ & 11 & $\begin{array}{l}c(3 \\
2.95\end{array}$ & $\begin{array}{l}b(2) \\
2.93\end{array}$ \\
\hline \begin{tabular}{l|l|} 
No. 8 & Liver power \\
(6575) & Body weight (in kilos)
\end{tabular} & $\begin{array}{l}c(3) \\
2.50\end{array}$ & $\begin{array}{l}c(3) \\
2.38\end{array}$ & $\begin{array}{l}d(4) \\
2.35\end{array}$ & 10 & $\begin{array}{l}a(4) \\
2.37\end{array}$ & $\begin{array}{l}f(6) \\
2.35\end{array}$ & $\begin{array}{l}2.40 \\
2.40\end{array}$ & 14 & $\begin{array}{l}b(2 \\
2.35\end{array}$ & $\begin{array}{l}f^{\prime}(6) \\
2.38\end{array}$ \\
\hline \begin{tabular}{l|l|} 
No.9 & Liver power \\
$(6576)$ & Body weight (in kilos)
\end{tabular} & $\begin{array}{l}f(6) \\
2.70\end{array}$ & $\begin{array}{l}f(6) \\
2.65\end{array}$ & $\begin{array}{l}b .(2) \\
2.67\end{array}$ & 14 & $\begin{array}{l}d(4) \\
2.60\end{array}$ & $\begin{array}{l}7(6) \\
2.50\end{array}$ & $\begin{array}{l}d(4) \\
2.45\end{array}$ & 14. & $\begin{array}{ll}b 2 \\
2.42\end{array}$ & 2.50 \\
\hline \begin{tabular}{|l|l} 
No.10 & Liver power \\
$(6577)$ & Body weight (in kilos)
\end{tabular} & $\begin{array}{l}d(4) \\
2.70 \\
\end{array}$ & $\begin{array}{l}b(2) \\
2.65\end{array}$ & $\begin{array}{l}f(6) \\
2.67\end{array}$ & 12 & $\begin{array}{l}e(5) \\
3.00\end{array}$ & $\begin{array}{l}c(3) \\
2.80 \\
\end{array}$ & $\begin{array}{l}f(6) \\
2.70\end{array}$ & 14 & $\begin{array}{l}c(3) \\
2.75\end{array}$ & $\begin{array}{l}b(2) \\
2.70 \\
\end{array}$ \\
\hline $\begin{array}{l}\text { No.11 Liver power } \\
\text { (6578) Body weight (in kilos) }\end{array}$ & $\begin{array}{l}b(2) \\
3.13\end{array}$ & $\begin{array}{l}f(6) \\
3.15\end{array}$ & $\begin{array}{l}c(3) \\
3.25\end{array}$ & I1 & $\begin{array}{l}f(6) \\
3.00\end{array}$ & $\begin{array}{l}f(6) \\
3.00\end{array}$ & $\begin{array}{l}f(6) \\
3.05\end{array}$ & 18 & $+2 . \mathrm{II}$ & \\
\hline \begin{tabular}{l|l} 
No. 12 & Liver power \\
$(6579)$ & Body weight (in
\end{tabular} & $\begin{array}{l}c(3) \\
2.60\end{array}$ & $\begin{array}{l}b(2) \\
2.60 \\
\text { Av }\end{array}$ & $\begin{array}{c}c(3) \\
2.67 \\
\text { rage }\end{array}$ & 8 & $\begin{array}{l}f^{\prime}(6) \\
2.70\end{array}$ & $\begin{array}{l}b(2) \\
2.60 \\
\text { Ave }\end{array}$ & $\begin{array}{l}d(4) \\
2.68 \\
\text { rage }\end{array}$ & 12 & $\begin{array}{r}d(4) \\
2.57 \\
\text { A }\end{array}$ & $\begin{array}{l}b(2) \\
2.60 \\
\text { erage }\end{array}$ \\
\hline
\end{tabular}


3.

ment in winter.

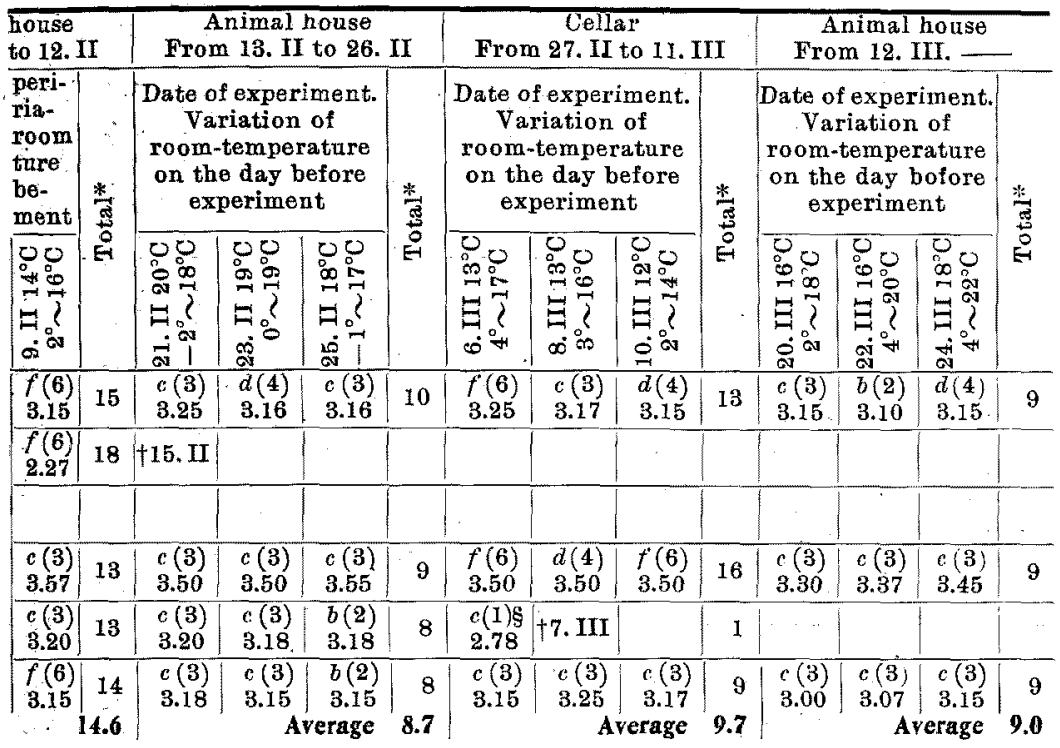

ment in winter.

\begin{tabular}{|c|c|c|c|c|c|c|c|c|c|c|c|c|c|}
\hline \multicolumn{2}{|c|}{$\begin{array}{l}\text { house } \\
\text { to } 12 . \mathrm{II}\end{array}$} & \multicolumn{4}{|c|}{$\begin{array}{l}\text { Cellar } \\
\text { From 13. II to 26. II }\end{array}$} & \multicolumn{4}{|c|}{$\begin{array}{l}\text { Animal house } \\
\text { From 27. II to I1. III }\end{array}$} & \multicolumn{4}{|c|}{$\begin{array}{c}\text { Cellar } \\
\text { From 12. III to } 25 \text {. III }\end{array}$} \\
\hline $\begin{array}{l}\text { peri- } \\
\text { ria- } \\
\text { room } \\
\text { ture } \\
\text { be- } \\
\text { ment }\end{array}$ & \multirow{2}{*}{ 齿 } & \multicolumn{3}{|c|}{$\begin{array}{l}\text { Date of experiment. } \\
\text { Variation of } \\
\text { room-temperature } \\
\text { on the day before } \\
\text { experiment }\end{array}$} & \multirow{2}{*}{ 螜 } & \multicolumn{3}{|c|}{$\begin{array}{l}\text { Date of experiment. } \\
\text { Variation of } \\
\text { room-temperature } \\
\text { on the day before } \\
\text { experiment }\end{array}$} & \multirow{2}{*}{ 空 } & \multicolumn{3}{|c|}{$\begin{array}{l}\text { Date of experiment. } \\
\text { Variation of } \\
\text { room-temperature } \\
\text { on the day before } \\
\text { experiment }\end{array}$} & \multirow{2}{*}{$\begin{array}{l}\text { 壱 } \\
\text { 志 } \\
\text { 品 }\end{array}$} \\
\hline 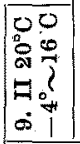 & & 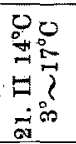 & 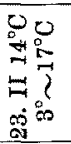 & 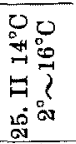 & & $\begin{array}{l}0 \\
0 \\
0 \\
0 \\
0 \\
=1 \\
0 \\
0 \\
0\end{array}$ & 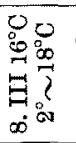 & $\begin{array}{l}0 \\
0 \\
0 \\
0 \\
B \\
0 \\
0 \\
0\end{array}$ & & 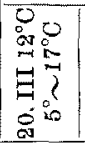 & 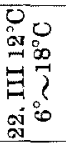 & 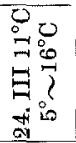 & \\
\hline $\begin{array}{l}c(3) \\
2.97\end{array}$ & 8 & $\begin{array}{l}f(6) \\
3.00\end{array}$ & $\begin{array}{l}d(4) \\
2.95\end{array}$ & $\begin{array}{l}f(6) \\
2.95\end{array}$ & 16 & $\begin{array}{l}\text { c. (3) } \\
2.80\end{array}$ & $\begin{array}{l}c(3) \\
2.95\end{array}$ & $\begin{array}{l}c(3) \\
2.90\end{array}$ & 9 & $\begin{array}{l}c(3) \\
2.95\end{array}$ & $\begin{array}{l}d(4) \\
2.90\end{array}$ & $\begin{array}{l}f(6) \\
2.85\end{array}$ & 13 \\
\hline $\begin{array}{l}f(6) \\
2.40\end{array}$ & 14 & $\begin{array}{l}f(6) \\
2.35\end{array}$ & $\begin{array}{l}f(6) \\
2.30\end{array}$ & $\begin{array}{l}(5) \\
2.34\end{array}$ & 17 & $\begin{array}{l}d(4) \\
2.40\end{array}$ & $\begin{array}{l}c(3) \\
2.50\end{array}$ & $\begin{array}{l}c(3) \\
2.67\end{array}$ & 10 & $\begin{array}{l}c(3) \\
2.55\end{array}$ & $\begin{array}{l}b(2) \\
2.45\end{array}$ & $\begin{array}{l}d(4) \\
2.45\end{array}$ & 9 \\
\hline $\begin{array}{l}b(2) \\
2.45\end{array}$ & 6 & $\begin{array}{l}f(6) \\
2.50\end{array}$ & $\begin{array}{l}e(5) \\
2.50\end{array}$ & $\begin{array}{l}f(6) \\
2.50\end{array}$ & 17 & $\begin{array}{l}b(2) \\
2.50\end{array}$ & $\begin{array}{l}b(2) \\
2.45\end{array}$ & $\begin{array}{l}c(3) \\
2.55\end{array}$ & 7 & $\begin{array}{l}f(6) \\
2.63\end{array}$ & $\begin{array}{l}b(2) \\
2.53\end{array}$ & $\begin{array}{l}f(6) \\
2.50\end{array}$ & 14 \\
\hline $\begin{array}{l}c(3) \\
2.73\end{array}$ & 8 & $\begin{array}{l}t^{2}(6) \\
2.50\end{array}$ & $\begin{array}{l}a(4) \\
2.60\end{array}$ & $\begin{array}{l}b(2) \\
2.65\end{array}$ & 12 & $\begin{array}{l}b(2) \\
2.50 \\
\end{array}$ & $\begin{array}{l}c(3) \\
2.45\end{array}$ & $\begin{array}{l}c(3) \\
2.50\end{array}$ & 8 & $\begin{array}{l}f(6) \\
2.54\end{array}$ & $\begin{array}{l}f(6) \\
2.50\end{array}$ & $\begin{array}{l}d(4) \\
2.56\end{array}$ & 16 \\
\hline $\begin{array}{l}b(2) \\
2.55\end{array}$ & $\begin{array}{r}8 \\
8.8\end{array}$ & $\begin{array}{l}d(4) \\
2.68\end{array}$ & $\begin{array}{r}r(3) \\
2.45 \\
\mathrm{Av}\end{array}$ & $\begin{array}{l}d(4) \\
2.55 \\
\text { age }\end{array}$ & $\begin{array}{r}11 \\
14.6\end{array}$ & 28.11 & & rage & 5.7 & & & age & 13.0 \\
\hline
\end{tabular}


Table

(A)

Result of the 1st experi-

\begin{tabular}{|c|c|c|c|c|c|c|c|c|}
\hline \multicolumn{2}{|c|}{ Where animals were kept } & \multicolumn{4}{|c|}{$\begin{array}{c}\text { Cellar } \\
\text { From 19. VI to I. VII }\end{array}$} & \multicolumn{3}{|c|}{$\begin{array}{r}\text { Animal house } \\
\text { From } 2 \text {. VII to } 15 .\end{array}$} \\
\hline \multirow{2}{*}{\multicolumn{2}{|c|}{ Number of rabbits }} & \multicolumn{3}{|c|}{$\begin{array}{c}\text { Date of experiment. } \\
\text { Variation of } \\
\text { room-temperature } \\
\text { on the day before } \\
\text { experiment } \\
\end{array}$} & \multirow{2}{*}{ 荡 } & \multicolumn{3}{|c|}{$\begin{array}{c}\text { Date of experiment. } \\
\text { Variation of } \\
\text { room-temperature } \\
\text { on the day before } \\
\text { experiment }\end{array}$} \\
\hline & & 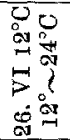 & 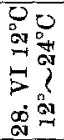 & {$\left[\begin{array}{l}0 \\
0 \\
0 \\
7 \\
0 \\
0 \\
0 \\
0 \\
0 \\
0 \\
0\end{array}\right.$} & & 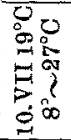 & 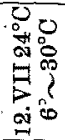 & 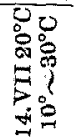 \\
\hline $\begin{array}{l}\text { No. } 1 \\
(6581)\end{array}$ & $\begin{array}{l}\text { Liver power } \\
\text { Body weight (in kilos) }\end{array}$ & $\begin{array}{l}f(6) \\
1.70\end{array}$ & $\begin{array}{l}f(6) \\
1.85\end{array}$ & $\begin{array}{l}f(6) \\
1.65\end{array}$ & 18 & $\begin{array}{l}d(4) \\
1.65\end{array}$ & $\begin{array}{l}d(4) \\
1.65\end{array}$ & $\begin{array}{l}d(4) \\
1.65\end{array}$ \\
\hline $\begin{array}{l}\text { No. } 2 \\
(6582)\end{array}$ & $\begin{array}{l}\text { Liver power } \\
\text { Body weight (in kilos) }\end{array}$ & $\begin{array}{l}c(3) \\
1.50\end{array}$ & $\begin{array}{l}b(2) \\
1.60\end{array}$ & $\begin{array}{l}b(2) \\
1.58\end{array}$ & 7 & $\begin{array}{l}a(4) \\
1.82\end{array}$ & $\begin{array}{l}\bar{d}(4) \\
1.90\end{array}$ & $\begin{array}{l}f(6) \\
1.80\end{array}$ \\
\hline $\begin{array}{l}\text { No. } 3 \\
\langle 6583\}\end{array}$ & $\begin{array}{l}\text { Liver power } \\
\text { Body weight (in kilos) }\end{array}$ & $\begin{array}{l}e(5) \\
1.45 \\
\end{array}$ & $\begin{array}{l}c(3) \\
1.45 \\
\end{array}$ & $\begin{array}{l}f(6) \\
1.35\end{array}$ & 14 & $\begin{array}{l}f(6) \\
1.60\end{array}$ & $\begin{array}{l}f(6) \\
1.60\end{array}$ & $\begin{array}{l}c(3) \\
1.60\end{array}$ \\
\hline $\begin{array}{l}\text { No. } 4 \\
(6584)\end{array}$ & $\begin{array}{l}\text { Liver power } \\
\text { Body weight (in kilos) }\end{array}$ & $\begin{array}{l}b(2) \\
1.75\end{array}$ & $\begin{array}{l}e(5) \\
1.75\end{array}$ & $\begin{array}{l}6(2) \\
1.65\end{array}$ & 9 & $\begin{array}{l}b(2) \\
1.80\end{array}$ & $\begin{array}{l}c(3) \\
1.75\end{array}$ & $\begin{array}{l}c(\mathrm{~B}) \\
1.80\end{array}$ \\
\hline $\begin{array}{l}\text { No. } 5 \\
(6586)\end{array}$ & $\begin{array}{l}\text { Liver power } \\
\text { Body weight (in kilos) }\end{array}$ & $\begin{array}{l}\bar{d}(4) \\
1.75\end{array}$ & $\begin{array}{l}t(8) \\
1.73\end{array}$ & $\begin{array}{l}c(3) \\
1.65\end{array}$ & 13 & $\begin{array}{l}b(2) \\
1.73\end{array}$ & $\begin{array}{l}d(4) \\
1.75\end{array}$ & $\begin{array}{l}6(2) \\
1.80\end{array}$ \\
\hline $\begin{array}{l}\text { No. } 6 \\
(6587)\end{array}$ & $\begin{array}{l}\text { Liver power } \\
\text { Body weight (in kilos) }\end{array}$ & $\begin{array}{l}c(3) \\
1.65\end{array}$ & $\begin{array}{l}d(4) \\
1.70\end{array}$ & \begin{tabular}{|c|}
$c(3)$ \\
1.65
\end{tabular} & $\begin{array}{l}10 \\
11.7\end{array}$ & $\begin{array}{l}c(3) \\
1.83\end{array}$ & $\begin{array}{l}c(3) \\
1.85\end{array}$ & $\begin{array}{l}c(3) \\
1.88 \\
\text { erage }\end{array}$ \\
\hline
\end{tabular}

Result of the 2nd experi-

\begin{tabular}{|c|c|c|c|c|c|c|c|c|}
\hline \multicolumn{2}{|c|}{ Where animals were kept } & & \multicolumn{3}{|c|}{$\begin{array}{c}\text { Animal house } \\
\text { to } 1 \text {. VII }\end{array}$} & \multicolumn{3}{|c|}{$\begin{array}{c}\text { Cellar } \\
\text { From 2. VII to } 25 .\end{array}$} \\
\hline \multirow{2}{*}{\multicolumn{2}{|c|}{ Number of rabbits }} & \multicolumn{3}{|c|}{$\begin{array}{l}\text { Date of experiment. } \\
\text { Variation of } \\
\text { room-temperature } \\
\text { on the day before } \\
\text { experiment }\end{array}$} & \multirow{2}{*}{ 粦 } & \multicolumn{3}{|c|}{$\begin{array}{l}\text { Date of experiment. } \\
\text { Variation of } \\
\text { room-temperature } \\
\text { on the day before } \\
\text { experiment }\end{array}$} \\
\hline & & 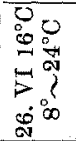 & 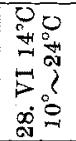 & $\begin{array}{l}0 \\
\infty \\
\infty \\
\infty \\
\infty \\
\infty \\
\infty \\
0 \\
0 \\
0\end{array}$ & & 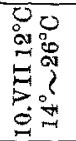 & 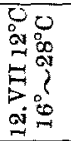 & $\begin{array}{l}0 \\
0 \\
0 \\
+1 \\
0 \\
0 \\
0 \\
0 \\
0 \\
0 \\
0\end{array}$ \\
\hline $\begin{array}{l}\text { No. } 7 \\
(6590)\end{array}$ & $\begin{array}{l}\text { Liver power } \\
\text { Body weight (in kilos) }\end{array}$ & $\begin{array}{l}b(2) \\
1.80\end{array}$ & $\begin{array}{l}d(4) \\
1.75\end{array}$ & $\begin{array}{l}c(3) \\
1.80\end{array}$ & 9 & $\begin{array}{l}d(4) \\
1.88\end{array}$ & $\begin{array}{l}d(4) \\
1.90\end{array}$ & $\begin{array}{l}d(4) \\
1.70\end{array}$ \\
\hline $\begin{array}{l}\text { No. } 8 \\
(6591)\end{array}$ & $\begin{array}{l}\text { Liver power } \\
\text { Body weight (in kilos) }\end{array}$ & $\begin{array}{l}a(4) \\
1.87\end{array}$ & $\begin{array}{l}d(4) \\
1.95\end{array}$ & $\begin{array}{l}f(6) \\
2.00\end{array}$ & 14 & $\begin{array}{l}d(4) \\
2.10\end{array}$ & $\begin{array}{l}e(5) \\
1.95\end{array}$ & $\begin{array}{l}f(6) \\
1.85\end{array}$ \\
\hline $\begin{array}{l}\text { No.9 } \\
(6592) \\
\end{array}$ & $\begin{array}{l}\text { Liver power } \\
\text { Body weight (in kilos) }\end{array}$ & $\begin{array}{l}b(2) \\
2.30\end{array}$ & $\begin{array}{l}f(6) \\
2.30\end{array}$ & $\begin{array}{l}f(6) \\
2.25\end{array}$ & 14 & $\begin{array}{l}b(2) \\
2.35\end{array}$ & $\begin{array}{l}f(6) \\
2.40\end{array}$ & $\begin{array}{l}c(3) \\
2.35 \\
\end{array}$ \\
\hline $\begin{array}{l}\text { No. } 10 \\
(6598)\end{array}$ & $\begin{array}{l}\text { Liver power } \\
\text { Body weight (in kifos) }\end{array}$ & $\begin{array}{l}c(3) \\
2.87\end{array}$ & $\begin{array}{l}c(3) \\
2.90\end{array}$ & $\begin{array}{l}6(3) \\
2.83\end{array}$ & 9 & $\begin{array}{l}d(4) \\
2.67\end{array}$ & $\begin{array}{l}d(4) \\
2.70\end{array}$ & $\begin{array}{l}f(6) \\
2.85\end{array}$ \\
\hline $\begin{array}{l}\text { No. } 11 \\
(6594)\end{array}$ & $\begin{array}{l}\text { Liver power } \\
\text { Body weight (in kilos) }\end{array}$ & $\begin{array}{l}c(3) \\
2.40\end{array}$ & $\begin{array}{l}b(2) \\
2.30\end{array}$ & $\mid \begin{array}{c}b(2) \\
2.25 \\
\text { Average }\end{array}$ & $\begin{array}{c}7 \\
10.6\end{array}$ & $\begin{array}{l}f(6) \\
2.43\end{array}$ & $\begin{array}{l}d(4) \\
2.40\end{array}$ & $\begin{array}{c}f(6) \\
2.35 \\
\text { Average }\end{array}$ \\
\hline
\end{tabular}


4.

ment in summer.

\begin{tabular}{|c|c|c|c|c|c|c|c|c|c|c|c|c|}
\hline VII & From & $\begin{array}{r}\mathrm{Ce} \\
6 . \mathrm{V}\end{array}$ & 29. & & From & $0 . \mathrm{V}$ & to & III & From & 12. V & to 2 & III \\
\hline$\frac{*}{z}$ & $\begin{array}{r}\text { Date } \\
\text { Voom } \\
\text { on th } \\
\text { ex }\end{array}$ & $\begin{array}{l}\text { mpe } \\
\text { may }\end{array}$ & $\begin{array}{l}\text { aent. } \\
\text { ff } \\
\text { bure } \\
\text { fore }\end{array}$ & $\frac{*}{7}$ & $\begin{array}{r}\text { Date } \\
\text { Va } \\
\text { room } \\
\text { on th } \\
\text { ex } \\
\end{array}$ & $\begin{array}{l}\text { iati } \\
\text { emp } \\
\text { day }\end{array}$ & $\begin{array}{l}\text { fure } \\
\text { fore } \\
t\end{array}$ & $\frac{*}{2}$ & $\begin{array}{r}\text { Date } \\
\mathrm{Va} \\
\text { room } \\
\text { on th } \\
\text { ex }\end{array}$ & $\begin{array}{l}\text { exp } \\
\text { iatio } \\
\text { emp } \\
\text { day }\end{array}$ & $\begin{array}{l}\text { nent. } \\
\text { of } \\
\text { ture } \\
\text { fore } \\
t\end{array}$ & 丞 \\
\hline Eे & 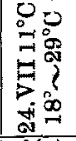 & 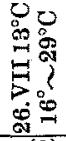 & 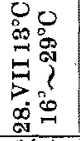 & : & $\begin{array}{r}0 \\
=0 \\
50 \\
50 \\
0 \\
0\end{array}$ & 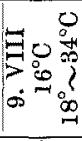 & 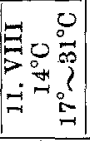 & है & 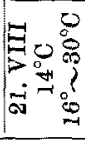 & 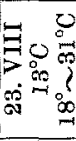 & 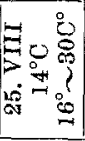 & है \\
\hline 12 & $\begin{array}{l}f(6) \\
1.93\end{array}$ & $\begin{array}{l}b(2) \\
1.92 \\
\end{array}$ & $\begin{array}{l}f^{\circ}(6) \\
1.95\end{array}$ & 14 & $\begin{array}{l}b(2) \\
2.00\end{array}$ & $\begin{array}{l}d(4) \\
2.02 \\
\end{array}$ & $\begin{array}{l}d(4) \\
2.05\end{array}$ & 10 & $\begin{array}{l}f^{2}(6) \\
2.20\end{array}$ & $\begin{array}{l}b(2) \\
2.25\end{array}$ & $\begin{array}{l}b(2) \\
2.18 \\
\end{array}$ & 10 \\
\hline 14 & $\begin{array}{l}f(\mathbb{1}) \S \\
1.85\end{array}$ & $\begin{array}{l}125 . \\
\text { VII }\end{array}$ & & 1 & & & & & & & & \\
\hline 15 & $\begin{array}{l}b(2) \\
1.85\end{array}$ & $\begin{array}{l}c(3) \\
1.87\end{array}$ & $\begin{array}{l}c(3) \\
1.95\end{array}$ & 8 & $\begin{array}{l}b(2) \\
2.28\end{array}$ & $\begin{array}{l}c(3) \\
2.29\end{array}$ & $\begin{array}{l}c(3) \\
2.30\end{array}$ & 8 & $\begin{array}{l}c(3) \\
2.35\end{array}$ & $\begin{array}{l}f(6) \\
2.44\end{array}$ & $\begin{array}{l}d(4) \\
2.44\end{array}$ & 13 \\
\hline 8 & $\begin{array}{l}b(2) \\
1.92\end{array}$ & $\begin{array}{l}e(5) \\
1.92\end{array}$ & $\begin{array}{l}d(4) \\
1.70\end{array}$ & 11 & $\begin{array}{l}b(2) \\
1.95\end{array}$ & $\begin{array}{l}b(2) \\
1.93\end{array}$ & $\begin{array}{l}b(2) \\
1.95\end{array}$ & 6 & $\begin{array}{l}b(2) \\
2.00\end{array}$ & $\begin{array}{l}b(2) \\
1.98\end{array}$ & $\begin{array}{l}e(5) \\
1.93\end{array}$ & $\theta$ \\
\hline - 8 & $\begin{array}{l}f(6) \\
1.93\end{array}$ & $\begin{array}{l}f(6) \\
1.95\end{array}$ & $\begin{array}{l}f(6) \\
1.98\end{array}$ & 18 & $\begin{array}{l}b(2) \\
2.00\end{array}$ & $\begin{array}{l}b(2) \\
2.05\end{array}$ & $\begin{array}{l}b(2) \\
2.05\end{array}$ & 6 & $\begin{array}{l}d(4) \\
2.21\end{array}$ & $\begin{array}{l}f(6) \\
2.20\end{array}$ & $\begin{array}{l}\text { c(3) } \\
2.24\end{array}$ & 13 \\
\hline $\begin{array}{c}9 \\
11.0\end{array}$ & $\begin{array}{l}c(3) \\
2.16\end{array}$ & $\begin{array}{l}f(6) \\
2.20\end{array}$ & $\begin{array}{l}f(6) \\
2.22 \\
\text { verage }\end{array}$ & $\begin{array}{r}15 \\
11 .\end{array}$ & $\begin{array}{l}b(2) \\
2.45\end{array}$ & $\begin{array}{l}b(2) \\
2.33\end{array}$ & \begin{tabular}{|c|}
$b(2)$ \\
2.52 \\
Average
\end{tabular} & $\begin{array}{l}6 \\
7.2\end{array}$ & $\begin{array}{l}f(6) \\
2.60\end{array}$ & $\begin{array}{l}f(6) \\
2.77\end{array}$ & \begin{tabular}{|l|}
$b(2)$ \\
2.64 \\
Average
\end{tabular} & $\begin{array}{l}14 \\
11.8\end{array}$ \\
\hline
\end{tabular}

ment in summer.

\begin{tabular}{|c|c|c|c|c|c|c|c|c|c|c|c|c|}
\hline \multirow{3}{*}{ 䄅 } & \multicolumn{4}{|c|}{$\begin{array}{l}\text { Animal house } \\
\text { From } 16 \text {. VII to } 29 \text {. VII }\end{array}$} & \multicolumn{4}{|c|}{ From 30. VII to 12. VIII } & \multicolumn{4}{|c|}{$\begin{array}{l}\text { Animal house } \\
\text { From 13. VIII }\end{array}$} \\
\hline & \multicolumn{3}{|c|}{$\begin{array}{c}\text { Date of experiment. } \\
\text { Variation of } \\
\text { room-temperature } \\
\text { on the day before } \\
\text { experiment }\end{array}$} & \multirow{2}{*}{ 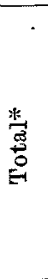 } & \multicolumn{3}{|c|}{$\begin{array}{l}\text { Date of experiment. } \\
\text { Variation of } \\
\text { room-temperature } \\
\text { on the day before } \\
\text { experiment }\end{array}$} & \multirow{2}{*}{ 装 } & \multicolumn{3}{|c|}{$\begin{array}{l}\text { Date of experiment. } \\
\text { Variation of } \\
\text { room-temperature } \\
\text { on the day before } \\
\text { experiment }\end{array}$} & \multirow{2}{*}{ 楚 } \\
\hline & $\begin{array}{l}0 \\
0 \\
0 \\
0 \\
0 \\
0 \\
0 \\
0 \\
0 \\
0 \\
0\end{array}$ & 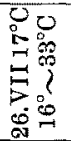 & {$\left[\begin{array}{l}0 \\
0 \\
0 \\
0 \\
0 \\
0 \\
0 \\
0 \\
0 \\
0 \\
0 \\
0 \\
0 \\
0\end{array}\right.$} & & 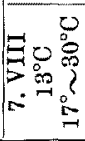 & 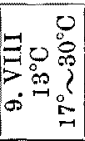 & 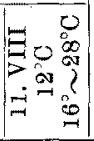 & & 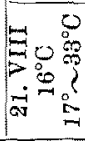 & 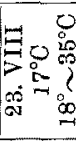 & $\left|\begin{array}{lll}\beta & & 0 \\
\beta & 0 & 0 \\
\beta & 0 & 0 \\
0 & 0 & 0 \\
0 & & 0 \\
0 & 0\end{array}\right|$ & \\
\hline 12 & $\begin{array}{l}d(4) \\
1.80\end{array}$ & $\begin{array}{l}c(3) \\
1.76\end{array}$ & $\begin{array}{l}b(2) \\
1.85\end{array}$ & 9 & $\begin{array}{l}e(3) \\
1.98\end{array}$ & $\begin{array}{l}f(6) \\
2.00\end{array}$ & $\begin{array}{l}d(4) \\
2.00\end{array}$ & 13 & $\begin{array}{l}b(2) \\
2.00\end{array}$ & $\begin{array}{l}b(2) \\
2.10\end{array}$ & $\begin{array}{l}b(2) \\
2.10\end{array}$ & 6 \\
\hline 15 & $\begin{array}{l}d(4) \\
2.05\end{array}$ & $\begin{array}{l}d(4) \\
2.10\end{array}$ & $\begin{array}{l}b(2) \\
2.15\end{array}$ & 10 & $\begin{array}{l}d(4) \\
2.50\end{array}$ & $\begin{array}{l}a(I) \\
2.40\end{array}$ & $\begin{array}{c}+9 . \\
\text { VIII }\end{array}$ & 5 & & & & \\
\hline 11 & $\begin{array}{l}f(6) \\
2.35\end{array}$ & $\begin{array}{l}b(2) \\
2.45\end{array}$ & $\begin{array}{l}b(2) \\
2.43\end{array}$ & 10 & $\begin{array}{l}b(2) \\
2.57\end{array}$ & $\begin{array}{l}d(4) \\
2.47\end{array}$ & $\begin{array}{l}d(4) \\
2.60\end{array}$ & 10 & $\begin{array}{l}d(4) \\
2.50\end{array}$ & $\begin{array}{l}a(1) \\
2.45\end{array}$ & $\begin{array}{r}+23 . \\
\text { VIII } \\
\end{array}$ & 5 \\
\hline 13 & $\begin{array}{l}f(6) \\
2.70\end{array}$ & $\begin{array}{l}e(5) \\
2.85\end{array}$ & $\begin{array}{l}0(3) \\
2.95\end{array}$ & 14 & $\begin{array}{l}e(5) \\
2.90\end{array}$ & $\begin{array}{l}a(1) \\
2.60\end{array}$ & $\begin{array}{l}+9 . \\
\text { VIII }\end{array}$ & 6 & & & & \\
\hline $\begin{array}{l}16 \\
13.4\end{array}$ & $\begin{array}{l}d(4) \\
2.60\end{array}$ & $\begin{array}{l}a(1) \\
2.65\end{array}$ & $\begin{array}{l}+27 . \\
\text { VII }\end{array}$ & 5 & & & & & & & & \\
\hline
\end{tabular}




\section{Comment.}

First let us describe what we deem ideal for the investigation of the present problem, then we shall proceed to the result we have obtained.

1. The seasons ideal for the present work.

The situation here is not ideal, because the summer ideal for our purpose, for instance, should be a very long one, at least half a year long. Of course, it should be constantly hot. Our summer is too short for the purpose. And rabbits should be "acclimatized" in the cellar or in the animal house at least for one month* instead of for one week as in our actual experiment.

2. The cellar and the animal house ideal for the present work.

They should of course be under exactly the same conditions except that the former is under the ground and the latter is on $\mathrm{it}$, and the rooms should be capable of being heated or cooled artificially and uniformally to be ideal-for our purpose. We used just the animal house, which is a section of this Laboratory, where we feel very hot in day time in summer, and very cold at night in winter. In the cellar we feel the heat or coldness somewhat less markedly.

3. The longevity of animals.

Rabbits ideal for the present work should be of much longer life than the usual period, as then we should be able to repeat the ammonium injection with a much longer interval, and repeat the experiment with one and the same animal for several years. With so many injections as in our actual experiment, they were impaired not unremarkably. One of us, $\mathrm{Sato}_{2}{ }^{2)}$ has the experience of about 120 ammonia injections in a single rabbit, but the rabbit had repeated injections of yakriton at the same time, otherwise it could not have outlived even half as many ammonium injections.

Now as to the actual result:

1. As stated above, our rabbits had too short a time for " acclimatisation." This is the reason why the actual result was not so significant as in our presumption.

* S. Kuribayashi (one of us) reported that a change of blood picture of rabbit, especially nuclear shift, produced by ammonia injection, was restored for about 4 weeks to what it had been before that injection. Tohoku J. Exp. Med., 1939., 36, 398.

2) A. Sato, Tohoku J. Exp. Med., 1928, 11, 272. 
2. The result should have been most remarkable in summer, but the actual result was not so. This was highly probably due to the condition that in warm weather the recovery from ammonia takes a much longer time. The two series of experiments in summer will show this, especially the 2nd experiment in summer. The 2nd point of $\mathrm{C}$ became lower than the preceding point $\mathrm{A}$. This was the only exception in the whole series of our present experiments. Besides, the number of rabbits that succumbed to the poisoning was remarkably larger in summer, which shows eloquently the lowering of ammonia detoxicating liver power in hot weather. The lowering was thus the most remarkable in summer.*

Cause for the lowering of the detoxicating liver power: The cause must most probably be heat. As stated above, the result apparently less conspicuous in summer was really due to the condition that the injury in summer was especially remarkable, and that the recovery from it occurred only slowly. The rather conspicuous result in autumn shows that the different response of one and the same animal toward ammonia was due to the difference of temperature between the cellar and the animal house, even in autumn. But whether the cause was the difference of temperature or the difference of the fluctuation of the temperature could not be decided by the present experiment. Further, it should be taken into account that the difference between summer and autumn comprises, besides that of temperature and moisture, a number of other factors which only future climatology can reveal to us.

Injury of coldness :' Our experience with rabbits has been that the animals were apt to become weakened in summer. We prefer to make experiments concerning the detoxicating liver power in spring and autumn. How about the winter? It is probable that the detoxicating liver power will suffer in winter too through the injury may not be so great as in summer. Takamatsu ${ }^{3)}$ who devised a method of the liver function test with yakriton and adrenalin, reported that the detoxicating liver test was the weakest in summer, and that in winter it was weaker than in autumn. In our present experiment, the result of experiment in winter is to be understood in that sense: The 1st experiment in winter (Fig. 2) was not very significant.

* As to the lowering in winter see later.

3) A. Takamats $u$, Tohoku J. Exp. Med., 1936, 28, 349. 


\section{Conclusions.}

It is known especially among pediatricians that in summer the liver power is lowered. We tried io test the ammonium detoxicating liver power in rabbits using Sato and Sakurada's ${ }^{1)}$ liver function test. Generally, they showed a higher-classed liver power in a place of mild temperature, and a low-classed liver power in a place of severe temperature. And they showed that the liver power was injured most remarkably in summer. 